\title{
DESENVOLVIMENTO DE UM PROTOCOLO DE AVALIAÇÃO DOS DADOS DE TEMPERATURA REGISTRADOS POR ESTAÇÕES METEOROLÓGICAS AUTOMÁTICAS NO CEARÁ
}

\author{
DEVELOPMENT OF A PROTOCOL FOR THE EVALUATION OF TEMPERATURE \\ DATA REGISTERED BY AUTOMATIC WEATHER STATIONS IN CEARÁ
}

\author{
Sousa Filho ${ }^{1}$, M. R. P. \\ mariofilho17@gmail.com
}

\begin{abstract}
Resumo
A análise de dados tem fundamental importância nos estudos da Climatologia. Dar uma forma de tratamento desses dados também é importante, pois é através de uma metodologia para estudo desses dados é que as pesquisas irão se nortear. Esse trabalho é uma sugestão de estipulação das faixas de temperatura para o estado do Ceará em suas diversas regiões. Foram usados como base os dados mensais das Normais Climatológicas do INMET (Instituto Nacional de Meteorologia) de onze estações automáticas no estado. O uso dessas estações foi uma forma de especializar ao máximo as diferentes regiões do Ceará. Após as análises do limite inferior (mínimas) e superior (máximas) das normais, foram estipulados as temperaturas máximas absolutas e mínimas absolutas, baseadas nos desvios padrões dos limites inferior e superior das normais do INMET para estas estações. O registro que ficar acima ou abaixo dos limiares de temperaturas máximas absolutas e mínimas absolutas, pode ser considerado um dado extremamente raro (de algum evento meteorológico anômalo) ou a indicação de que a estação automática necessita de manutenção. Essa é uma proposta para filtragem de dados de temperatura pelo usuário, ou por quem irá fornecê-los, além de um indicativo de reparos em estações automáticas.
\end{abstract}

Palavras-chave: intervalos, temperatura, Ceará.

\begin{abstract}
Data analysis has fundamental importance in studies of Climatology. Take a form of treatment of the data is also important because it is through a methodology for the study of these data is that the research will be guided. This work is a stipulation of the temperature for the state of Ceará in its various regions. Were used the basis of the data of the monthly Climatological Normals of INMET (National Institute of Meteorology) of eleven automatic stations in the state. The use of these stations was a way to cover the most different regions of Ceará. After the analysis of the lower bound (minimal) and higher (maximum) of normal, were stipulated absolute maximum temperatures and absolute minimum, based on the standard deviations of the lower and upper bounds of normal INMET to these stations. The registry be above or below the range of absolute maximum and absolute minimum temperatures, can be considered a given extremely rare (some anomalous meteorological event) or an indication of the automatic station needs maintenance. This is a proposal for temperature data filtering by user, or who will provide them, in addition to a call-to-repair in automatic stations.
\end{abstract}

Keywords: ranges, temperature, Ceará.

\section{INTRODUÇÃO}

O tratamento das informações meteorológicas é de suma importância tanto para a sociedade, quanto para a comunidade científica. Monitorar a dinâmica atmosférica é importante para dar seguimento a vários estudos técnicos (energia, agricultura, clima urbano, etc) e que, por ventura, farão com que haja uma melhor gestão sócio espacial, desde a menor localidade ao maior centro urbano.

A Manipulação de dados é importante para a elaboração de projetos e execução destes. É essencial que tais registros tenham qualidade, para que de fato se possam realizar as atividades mais 
adequadamente. Dessa forma, a metodologia proposta é uma tentativa de estabelecimento de limiares (faixas ou intervalos) para os registros de temperatura no estado do Ceará. É uma forma de criação de um protocolo de análise desses registros, para que os dados usados sejam apresentados de uma forma mais correta, evitando equívocos durante seu uso.

Essa pesquisa é parte de um projeto feito pela Funceme (Fundação Cearense de Meteorologia e Recursos Hídricos) intitulado "Desenvolvimento de um Protocolo de Avaliação dos Dados Coletados por Plataformas Automáticas de Coleta de Dados”, realizado em 2013, no qual foram desenvolvidos limiares de temperatura, umidade, pressão atmosférica, pluviometria, radiação solar, velocidade e direção do vento, mas, que no presente artigo será abordado a metodologia de como foram elaborados as faixas para temperatura.

Os registros atmosféricos são obtidos através de estações meteorológicas chamadas pela Funceme de PCD's (Plataforma de Coleta de Dados), e foram as séries históricas dessas estações usados durante os estudos, nos quais se puderam observar o ritmo climático do estado, além de erros. As Normais Climatológicas do INMET (Instituto Nacional de Meteorologia) entre 1961 e 1990, cujas foram as últimas normais estabelecidas, também foram usadas para se ter como base o comportamento atmosférico cearense.

É importante salientar que essa metodologia vale para qualquer estação automática, Datalogger, Termo-Higrômetro ou qualquer outro aparelho que meça temperatura no estado do Ceará.

\section{METODOLOGIA}

A realização dessa pesquisa terá como alvo desenvolver um protocolo de avaliação dos dados de temperatura no estado do Ceará. Foram usadas as onze estações meteorológicas do INMET no estado para ter uma melhor espacialização das diversas regiões cearenses. Vale fazer uma observação quanto à região da Ibiapaba, pois a mesma não possui estação automática do INMET. Assim, os limiares da estação de Guaramiranga foram replicados para tal região. Esses procedimentos serão detalhados adiante.

No presente trabalho será apresentado a metodologia e um exemplo de como ficou o formato dos limiares de temperatura para o estado do Ceará, ou seja, será abordado os detalhes para a geração do protocolo de avaliação desses coletados por PCD’s, gerando assim uma forma de controle de qualidade dos dados coletados.

Foram elaborados os limiares de temperatura máxima, mínima, máxima absoluta e mínima absoluta. O dado que se apresentar além das faixas de máxima absoluta e mínima absoluta, pode ser um dado passível de erro (algum problema na calibração do sensor ou problemas na transmissão) ou um evento meteorológico extremamente raro. 
Foi atribuído às faixas de temperatura máxima e mínima, os limiares das Normais Climatológicas do INMET para o estado do Ceará, cujo estudo foi feito através da série de dados de onze estações no estado, no período compreendido entre 1961 e 1990, e que foram estabelecidos as temperaturas máximas e mínimas mensais de cada uma dessas onze estações. Estas Normais Climatológicas foram adquiridas na Funceme. A seguir seguem as tabelas mostrando as temperaturas:

\begin{tabular}{||c|c|c|c|c|c|c|c|c|c|c|c|c|c|c|}
\hline \multicolumn{10}{|c|}{ TEMPERATURA MAXIMA $\left.{ }^{\circ} \mathbf{C}\right)$} \\
\hline Nos. & Municipios & JAN & FEV & MAR & ABR & MAI & JUN & JUL & AGO & SET & OUT & NOV & DEZ & ANO \\
\hline 82784 & Barbalha & 32.1 & 30.9 & 30.3 & 30.1 & 30.0 & 28.4 & 29.8 & 31.7 & 33.3 & 34.1 & 33.9 & 33.1 & 31.5 \\
\hline 82777 & Campos Sales & 31.1 & 30.4 & 29.1 & 29.1 & 29.4 & 29.4 & 29.5 & 30.9 & 30.8 & 33.1 & 32.9 & 32.4 & 30.7 \\
\hline 82583 & Crateús & 32.5 & 32.0 & 30.7 & 30.6 & 29.2 & 31.1 & 31.9 & 32.4 & 34.6 & 35.4 & 35.2 & 33.8 & 32.5 \\
\hline 82397 & Fortaleza & 30.5 & 30.1 & 29.7 & 29.7 & 29.1 & 29.6 & 29.5 & 29.1 & 29.2 & 30.5 & 30.7 & 30.7 & 29.9 \\
\hline 82487 & Guaramiranga & 25.9 & 25.4 & 24.7 & 24.4 & 23.3 & 22.9 & 23.4 & 25.1 & 26.5 & 26.8 & 26.0 & 26.5 & 25.1 \\
\hline 82686 & Iguatu & 33.3 & 32.0 & 31.2 & 29.8 & 27.8 & 27.3 & 28.5 & 33.2 & 33.2 & 33.9 & 35.2 & 34.7 & 31.7 \\
\hline 82493 & Jaguaruana & 33.8 & 32.9 & 29.4 & 31.8 & 32.0 & 31.5 & 32.0 & 33.2 & 34.0 & 34.1 & 34.0 & 33.7 & 32.7 \\
\hline 82588 & Morada Nova & 35.1 & 33.8 & 32.3 & 31.9 & 31.9 & 31.8 & 32.6 & 34.2 & 35.3 & 36.1 & 34.5 & 35.7 & 33.8 \\
\hline 82586 & Quixeramobim & 33.3 & 32.5 & 31.5 & 30.8 & 29.1 & 30.3 & 30.9 & 32.8 & 34.1 & 34.7 & 34.7 & 34.4 & 32.5 \\
\hline 82392 & Sobral & 33.8 & 32.9 & 30.1 & 31.1 & 31.2 & 31.6 & 33.0 & 34.8 & 35.8 & 35.9 & 35.6 & 34.0 & 33.3 \\
\hline 82683 & Tauá & 33.1 & 31.1 & 30.1 & 30.3 & 30.3 & 30.5 & 30.7 & 32.3 & 33.4 & 32.2 & 32.1 & 33.5 & 31.6 \\
\hline \hline
\end{tabular}

Figura 1: normais de temperatura máxima nas estações do INMET no Ceará. Fonte: INMET (1991).

\begin{tabular}{|c|c|c|c|c|c|c|c|c|c|c|c|c|c|c|}
\hline \multicolumn{15}{|c|}{ TEMPERATURA MINIMA ( $\left.{ }^{\circ} \mathrm{C}\right)$} \\
\hline Nos. & Municípios & JAN & FEV & MAR & ABR & MAI & JUN & JUL & AGO & SET & OUT & NOV & DEZ & ANO \\
\hline 82784 & Barbalha & 21.2 & 21.1 & 20.9 & 21.2 & 20.6 & 19.3 & 19.1 & 18.1 & 20.1 & 21.1 & 21.8 & 21.7 & 20.5 \\
\hline 82777 & ampos Sales & 21.4 & 20.3 & 21.7 & 20.4 & 19.0 & 18.3 & 18.2 & 18.7 & 19.4 & 20.9 & 21.7 & 21.3 & 20.1 \\
\hline 82583 & ús & 21.9 & 21.1 & 21.3 & 20.0 & 20.9 & 19.8 & 18.8 & 19.1 & 22.0 & 22.0 & 22.0 & 22.7 & 21.0 \\
\hline 82397 & $\mathrm{za}$ & 24.7 & 23.2 & 23.8 & 23.4 & 23.4 & 22.1 & 21.8 & 22.6 & 23.4 & 24.5 & 24.4 & 24.6 & 23.5 \\
\hline 82487 & Guara & 17.8 & 18.2 & 19.1 & 18.3 & 18.1 & 18.8 & 16.3 & 1 & 16.9 & 17.5 & 7.8 & 18.9 & 17.8 \\
\hline 86 & & 22.5 & 22.8 & 20.8 & 21.6 & 21.8 & 21.7 & 20.2 & .1 & 22.0 & 2.7 & 3.2 & 23.5 & 20.3 \\
\hline 82493 & 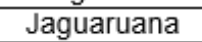 & 23.7 & 23.5 & 23.5 & 23.4 & 22.9 & 21.8 & 21.2 & .1 & 21.8 & 22.6 & 23.8 & 23.5 & 22.7 \\
\hline 82588 & ova & 22.3 & 23.5 & 23.0 & 21.8 & 22.6 & 21.4 & 20.8 & 0.6 & 21.4 & 22.1 & 0.8 & 22.9 & 21.9 \\
\hline 82586 & Quixer & 23.6 & 23.3 & 23.6 & 23.0 & 22.2 & 21.4 & 21.2 & 22.5 & 22.5 & 23.1 & 23.4 & 23.7 & 22.8 \\
\hline 82392 & Sobral & 23.6 & 22.0 & 22.5 & 22.6 & 21.3 & 21.5 & 21.2 & 21.4 & 21.5 & 21.5 & 22.0 & 23.3 & 22.0 \\
\hline 82683 & Tauá & 19.9 & 22.4 & 20.6 & 21.3 & 19.3 & 19.5 & 20.1 & 20.0 & 21.6 & 22.2 & 22.7 & 22.9 & 21.0 \\
\hline
\end{tabular}

Figura 2: normais de temperatura mínima nas estações do INMET no Ceará. Fonte: INMET (1991).

Para as faixas de temperatura que correspondem às máximas absolutas e mínimas absolutas, o parâmetro usado para estabelece-las foi definir os desvios padrões das temperaturas máximas e das mínimas das normais, e então, os valores encontrados foram usados para estabelecer os limiares de máximas absolutas e mínimas absolutas.

Por exemplo: após o cálculo do desvio padrão das temperaturas mínimas das normais para o mês de janeiro encontra-se um valor de $2,2^{\circ} \mathrm{C}$, logo, a faixa das temperaturas mínimas absolutas vai ser a temperatura mínima da normal do INMET subtraída a esse valor, ou seja, se a temperatura mínima normal em janeiro para Fortaleza é $24,7^{\circ} \mathrm{C}$, a faixa de temperaturas mínimas absolutas vai ficar entre 24,6 e $22,5^{\circ} \mathrm{C}\left(24,7-2,2^{\circ} \mathrm{C}\right)$. Abaixo segue um exemplo de um mês com as temperaturas máximas, mínimas, máximas absolutas e mínimas absolutas: 


\begin{tabular}{|c|c|c|c|c|}
\hline & \multicolumn{4}{|c|}{ NORIMAL - JANEIRO } \\
\hline & MÍN. ABSOLUTA & MÍNIMA & MÁXIMA & MÁX. ABSOLUTA \\
\hline Barbalha [CARIRI] & $19-21,1$ & 21,2 & 32,1 & $32,2-35,1$ \\
\hline Campos Sales [CHAP ARARIPE] & $19,2-21,3$ & 21,4 & 31,1 & $32,2-34,1$ \\
\hline Crateús [SERTÁO DE CRATEÚS] & $19,7-21,8$ & 21,9 & 32,5 & $32,6-35,5$ \\
\hline Fortaleza [FORTALEZA] & $22,5-24,6$ & 24,7 & 30,5 & $30,6-33,5$ \\
\hline Guaramiranga [BATURITÉ] & $15,6-17,7$ & 17,8 & 25,9 & $26-28,9$ \\
\hline Iguatu [IGUATU] & $20,3-22,4$ & 22,5 & 33,3 & $33,4-36,3$ \\
\hline Jaguaruana [BAIXO JAGUARIBE] & $21,5-23,6$ & 23,7 & 33,8 & $33,9-36,8$ \\
\hline Morada Nova [BAIXO JAGUARIBE] & $20,1-22,2$ & 22,3 & 35,1 & $35,2-38,1$ \\
\hline Quixeramobim [SERTÁO DE QUIXERAMOBIM] & $21,4-23,5$ & 23,6 & 33,3 & $33,4-36,3$ \\
\hline Sobral [SOBRAL] & $21,4-23,5$ & 23,6 & 33,8 & $33,9-36,8$ \\
\hline Tauá [ INHAMUNS] & $17,7-19,8$ & 19,9 & 33,1 & $33,2-36,1$ \\
\hline Săo Benedito [IBIAPABA] & $15,6-17,7$ & 17,8 & 25,9 & $26-28,9$ \\
\hline & DESVIO PAD. & 2,2 & 3,0 & \\
\hline
\end{tabular}

Figura 3: desvios padrões, máximas absolutas e mínimas absolutas para o mês de janeiro. Fonte: autor.

Em outro exemplo, podemos ver na imagem acima, que a temperatura máxima absoluta para Barbalha no mês de janeiro, é sua máxima mensal normal somada do desvio padrão das temperaturas máximas mensais das outras estações, ou seja, $32,1^{\circ} \mathrm{C}+3,0^{\circ} \mathrm{C}$ (desvio padrão) $=$ $35,1^{\circ} \mathrm{C}$. Logo, o limiar de temperaturas máximas mensais para o mês de janeiro em Barbalha vai ser entre 32,2 e $35,1^{\circ} \mathrm{C}$. Esse procedimento segue para as outras estações meteorológicas, lembrando que tal metodologia vale tanto para as máximas absolutas quanto mínimas absolutas.

A escolha do desvio padrão para estipular os limiares de temperaturas máximas absolutas e mínimas absolutas foi devido essa medida de dispersão ser caracterizada por analisar a variação dos valores em relação à média. Além disso, o desvio padrão fornece qual variável é mais regular (a que possui o menor desvio).

$$
S=\sqrt{\frac{\sum_{i=1}^{n}\left(x_{i}-x\right)^{2}}{n-1}}
$$

Figura 4: fórmula do desvio padrão: raiz quadrada da média aritmética dos quadrados dos desvios, ou seja: raiz quadrada da variância. Fonte: Freund (2006).

Quanto à regularidade das temperaturas das normais mensais, “... a dispersão de um conjunto de dados é pequena se os valores estão bem concentrados em torno da média, e é grande se os valores estão muito espalhados em torno da média" (FREUND, 2006). Pode-se observar que as temperaturas máximas possuem maior desvio padrão (maiores valores de dispersão) que as mínimas, fazendo com que elas sejam mais irregulares durante o ano no estado do Ceará: 


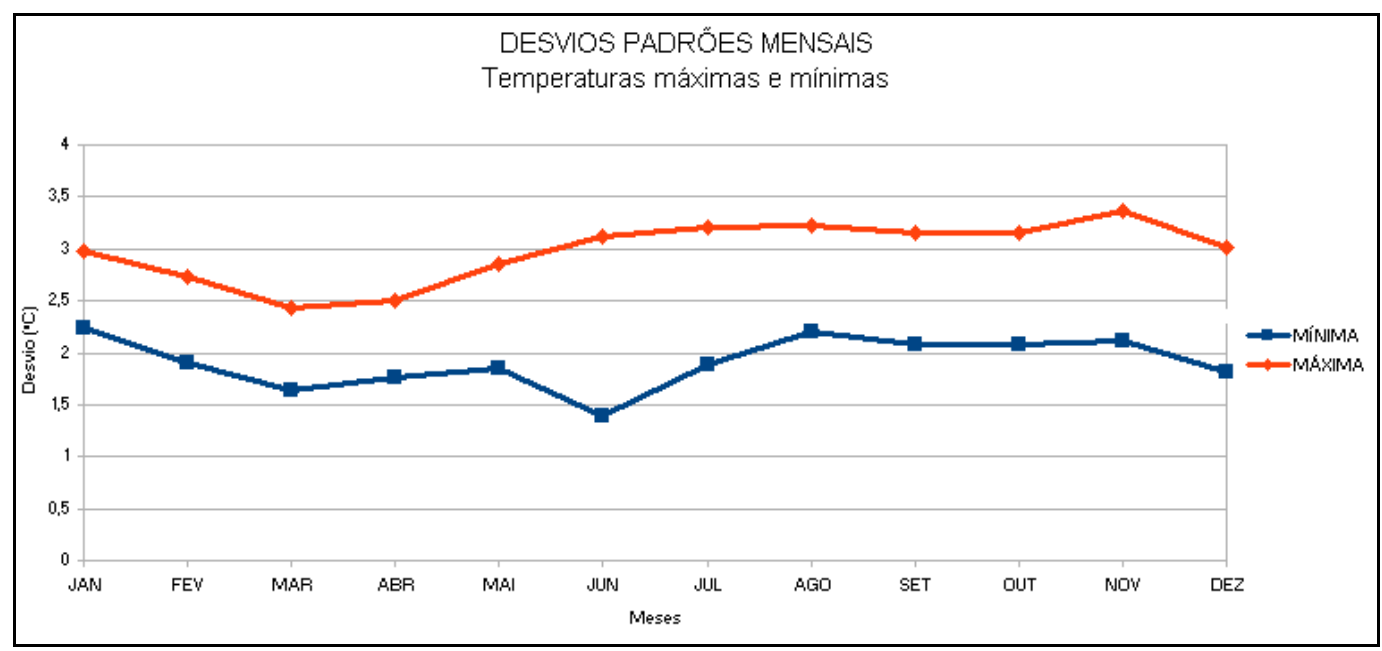

Figura 5: desvios padrões mensais. Note que a máxima tem maiores valores, sendo mais irregular. Fonte: autor.

\section{RESULTADOS E DISCUSSÃO}

Depois de estabelecidos os limiares de temperaturas máximas absolutas e mínimas absolutas, o que ficou acima ou abaixo destas, poderia ser considerado um dado extremamente raro (dias seguidos com pouca radiação solar, períodos em que o inverno se apresentasse com mais intensidade na região, etc.), ou passível de erro (vários registros além da faixa amarela estabelecida, problemas nos sensores, erros na transmissão do dado, etc.). Esses dados que ficassem acima ou abaixo dessa faixa, estão sinalizados com a cor vermelha.

Abaixo segue um exemplo de como ficaram estabelecidos os novos limiares de temperatura mensais, por região:

\begin{tabular}{|c|c|c|c|c|c|}
\hline \multicolumn{7}{|c|}{ TEMPERATURA JANEIRO $\left({ }^{\circ} \mathrm{C}\right)$} \\
\hline & ABAIXO DA MINIMA & MíN. ABSOLUTA & NORMAL & MÁX. ABSOLUTA & ACIMA DA MÁXIMA \\
\hline Barbalha [CARIRI] & $<18,9$ & $19-21,1$ & $21,2-32,1$ & $32,2-35,1$ & $>35,1$ \\
\hline Campos Sales [CHAP ARARIPE] & $<19,1$ & $19,2-21,3$ & $21,4-31,1$ & $32,2-34,1$ & $>34,1$ \\
\hline Crateús [SERTÄO DE CRATEÚS] & $<19,6$ & $19,7-21,8$ & $21,9-32,5$ & $32,6-35,5$ & $>35,5$ \\
\hline Fortaleza [FORTALEZA] & $<22,4$ & $22,5-24,6$ & $24,7-30,5$ & $30,6-33,5$ & $>33,5$ \\
\hline Guaramiranga [BATURITÉ] & $<15,5$ & $15,6-17,7$ & $17,8-25,9$ & $26-28,9$ & $>28,9$ \\
\hline Iguatu [IGUATU] & $<20,2$ & $20,3-22,4$ & $22,5-33,3$ & $33,4-36,3$ & $>36,3$ \\
\hline Jaguaruana [BAIXO JAGUARIBE] & $<21,4$ & $21,5-23,6$ & $23,7-33,8$ & $33,9-36,8$ & $>36,8$ \\
\hline Morada Nova [BAIXO JAGUARIBE] & $<20$ & $20,1-22,2$ & $22,3-35,1$ & $35,2-38,1$ & $>38,1$ \\
\hline Quixeramobim [SERTÄO DE QUIXERAMOBIM] & $<21,3$ & $21,4-23,5$ & $23,6-33,3$ & $33,4-36,3$ & $>36,3$ \\
\hline Sobral [SOBRAL] & $<21,3$ & $21,4-23,5$ & $23,6-33,8$ & $33,9-36,8$ & $>36,8$ \\
\hline Tauá [INHAMUNS] & $<17,6$ & $17,7-19,8$ & $19,9-33,1$ & $33,2-36,1$ & $>36,1$ \\
\hline São Benedito [IBIAPABA] & $<15,5$ & $15,6-17,7$ & $17,8-25,9$ & $26-28,9$ & $>28,9$ \\
\hline & & DP MiNIMA $=2,2$ & & DP MÁXIMA $=3,0$ & \\
\hline
\end{tabular}

Figura 6: limiares de temperatura do mês de janeiro. Fonte: autor.

Nesse exemplo para o mês de janeiro temos uma ideia do comportamento da temperatura em nosso estado, cujas temperaturas mínimas absolutas vão de $15,6^{\circ} \mathrm{C}$ e as máximas absolutas chegam aos $36,8^{\circ} \mathrm{C}$. Esses índices estão dentro da realidade de nosso estado, pois, valores por volta de $15^{\circ} \mathrm{C}$ é possível nos enclaves úmidos de nosso estado e registros acima dos $35^{\circ} \mathrm{C}$ é bastante comum na maior parte da região cearense, principalmente na região dos Inhamuns, Jaguaribana, de Sobral, Sertão Central, enfim, boa parte dos municípios que compõem a Depressão Sertaneja. 
Quanto aos limiares para o município de São Benedito (região da Ibiapaba), como dito anteriormente, tal localidade não possui estação meteorológica do INMET, mas tem características de altitude, latitude e pluviometria semelhantes ao município de Guaramiranga. Dessa forma, os intervalos propostos para Guaramiranga foram replicados para São Benedito. Abaixo segue uma tabela com o comparativo:

Tabela 01: Comparativo entre Guaramiranga e São Benedito. Fonte: IPECE (2013).

\begin{tabular}{|l|c|c|}
\hline \multicolumn{1}{|c|}{ VARIÁVEIS } & GUARAMIRANGA & SÃO BENEDITO \\
\hline Altitude (m) & 864,24 & 901,64 \\
\hline Latitude & $04^{\circ} 15^{\prime} 48^{\prime}{ }^{\prime} \mathrm{S}$ & $04^{\circ} 02^{\prime} 55^{\prime}{ }^{\prime} \mathrm{S}$ \\
\hline Pluviometria anual (mm) & 1737,5 & 1943,7 \\
\hline
\end{tabular}

É importante incluir a região da Ibiapaba nessa regionalização, pois a mesma é um enclave úmido (na parte que compõe o front da Cuesta) no estado, onde se desenvolve uma agricultura de frutas e flores para exportação, além de atividades turísticas, sendo um local de destaque no estado. Dessa forma, é importante entender o conhecimento da dinâmica térmica desse local e abrangê-lo no estudo.

\section{CONSIDERAÇÕES FINAIS}

A elaboração deste trabalho fará com que os dados das estações automáticas sejam indicados próprios para uso ou não, pois, após aplicação dessa metodologia, podem ser observados os dados corretos, defeituosos ou observar um evento extremamente raro que tenha acontecido.

É uma forma de controle de qualidade dos registros, para que se possa trabalhar com eles de uma forma mais segura, reduzindo o equívoco de usar dados passíveis de erros. A imagem abaixo ilustra essa questão:

\begin{tabular}{|c|c|c|}
\hline $\mathrm{N} / \mathrm{A}$ & & $32322 . \mathrm{TempAr}$ \\
\hline $02 / 08 / 2011$ & $00: 00: 00$ & 25.00 \\
\hline $02 / 08 / 2011$ & $03: 00: 00$ & 23.90 \\
\hline $02 / 08 / 2011$ & $06: 00: 00$ & 23.10 \\
\hline $02 / 08 / 2011$ & $09: 00: 00$ & 22.90 \\
\hline $02 / 08 / 2011$ & $12: 00: 00$ & 28.40 \\
\hline $02 / 08 / 2011$ & $15: 00: 00$ & 8.70 \\
\hline $02 / 08 / 2011$ & $18: 00: 00$ & 9.60 \\
\hline $02 / 08 / 2011$ & $21: 00: 00$ & 27.20 \\
\hline $02 / 09 / 2011$ & $00: 00: 00$ & 26.50 \\
\hline $02 / 09 / 2011$ & $03: 00: 00$ & 26.80 \\
\hline $02 / 09 / 2011$ & $06: 00: 00$ & 25.90 \\
\hline $02 / 09 / 2011$ & $09: 00: 00$ & 22.80 \\
\hline $02 / 09 / 2011$ & $12: 00: 00$ & 29.80 \\
\hline $02 / 09 / 2011$ & $15: 00: 00$ & 8.90 \\
\hline $02 / 09 / 2011$ & $18: 00: 00$ & 13.50 \\
\hline $02 / 09 / 2011$ & $21: 00: 00$ & 27.40 \\
\hline
\end{tabular}

Figura 7: temperatura na PCD de Caucaia (Funceme). Fonte: Funceme (2014) 
No exemplo acima observa-se que os registros apresentam problemas sempre às $15 \mathrm{e} 18 \mathrm{~h}$ (UTC), apresentando temperaturas que não correspondem com a realidade cearense, mas durante o restante do dia se apresentam normais. Isso é um indício de um problema na transmissão dos mesmos, pois, nos outros horários os dados se apresentam dentro da normalidade. Essa é uma forma de indicativo para início de investigação do que está acontecendo e corrigir o problema.

A proposta do estabelecimento desses limiares de temperatura para o estado do Ceará também possibilitará uma possível manutenção na rede de estações, pois será uma forma de alerta para identificação de problemas nos sensores ou na transmissão dos registros.

\section{REFERÊNCIAS}

DEPAM, Departamento Nacional de Meteorologia. Normais Climatológicas (1961 - 1990). Brasília, 1992.

FREUND, John E. Estatística Aplicada: Economia, Administração e Contabilidade. $11^{\mathrm{a}}$ ed. Porto Alegre: Bookman, 2006.

INMET, Instituto Nacional de Meteorologia. Normais Climatológicas 1961 - 1990.

IPECE, Instituto de Pesquisa e Estratégia Econômica do Ceará. Perfil Básico Municipal 2013 Guaramiranga. www.ipece.ce.gov.br/publicacoes/perfil_basico/pbm-2013/Guaramiranga.pdf, acesso em 05 de julho de 2016.

IPECE, Instituto de Pesquisa e Estratégia Econômica do Ceará. Perfil Básico Municipal 2013 São Benedito. http://www.ipece.ce.gov.br/publicacoes/perfil_basico/pbm-2013/Sao_Benedito.pdf, acesso em 05 de julho de 2016.

MARTINS, F.R.; GUARNIERI, R.A.; PEREIRA, E.B.; MANTELLI, S.; CHAGAS, R.C.; THOMAZ, C.; ANDRADE, E. Projeto SONDA-Rede Nacional de Estações para coleta de dados meteorológicos aplicados ao setor de energia. Anais do I Congresso Brasileiro de Energia Solar, Associação Brasileira de Energia Solar, Fortaleza, 2007.

SONDA, 2006: Sistema de Organização Nacional de Dados Ambientais - CPTEC/INPE. [online]: http://sonda.cptec.inpe.br/

WEBMET, 2006: Quality Assurance and Quality Control. The Meteorological Resource Center. [online]: http://www.webmet.com/met_monitoring/8.html

\section{AGRADECIMENTOS}

Agradeço à Funceme por ter cedido os dados e local para realização dessa pesquisa e à FUNCAP (Fundação Cearense de Apoio ao Desenvolvimento Científico e Tecnológico), órgão financiador do projeto.

Recebido em: 14/08/2016

Aceito para publicação em: 01/10/2016 